Reprod. Nutr. Dévelop., 1986, 26 (1 B), 263-264.

\title{
Estimation de la digestibilité de l'herbe pâturée par le mouton adulte au moyen de la lignine selon Christian. Essais préliminaires (1)
}

\author{
A. THÉWIS, E. FRANC̣OIS $\left({ }^{*}\right)$, F. RODRIGUEZ, N. BARTIAUX-THILL $\left({ }^{*}\right)$
}

Faculté des Sciences Agronomiques de l'Etat, Chaire de Productions Animales Passage des Déportés, 2, B 5800 Gembloux, Belgique

(*) Centre de Recherches Agronomiques de l'Etat

Avenue de la Faculté d'Agronomie, 22, B 5800 Gembloux, Belgique

Summary. Excellent agreement was found between digestibility coefficients of 3 forages determined in grazing sheep by independent methods : nitrogen and lignin faecal indexes and by the lignin ratio technique (œesophageal fistulas). Lignin determined by Christian's method, was previously shown to be indigestible in most cases.

Dans une étude effectuée sur des béliers alimentés au foin de prairie (Frère et al., 1981), nous avons montré que la lignine dosée par la méthode rapide de Christian (1971) possède les qualités requises d'un marqueur indigestible interne. Dans cette note, nous comparons les coefficients de digestibilité de l'herbe pâturée déterminés soit directement à partir des concentrations en azote ou en lignine fécale soit à partir de la lignine marqueur indigestible interne.

\section{Matériel et méthodes.}

Expérience 1. - Trois herbes de prairie naturelle de plaine et deux ray-grass italiens récoltés à des époques différentes ont fait l'objet d'un bilan alimentaire d'une durée de 7 à 10 jours sur un nombre de béliers adultes variant de 4 à 6 . La quantité de fourrage nécessaire pour chaque bilan a été fauchée en une seule fois et conservée à $-20^{\circ} \mathrm{C}$ jusqu'au moment de l'utilisation. Le niveau alimentaire a varié de 45 à $72 \mathrm{~g}$ de matière sèche $/ \mathrm{kg} \mathrm{P}^{0,75}$. Le taux de récupération fécale de la lignine et la digestibilité de la matière sèche (d M.S.) ont été déterminés.

Expérience 2. - Trois parcelles à base de ray-grass anglais ont été pâturées successivement par 8 béliers adultes, dont 2 étaient porteurs d'une canule œsophagienne et 4 étaient équipés d'un harnais permettant la récolte intégrale des fèces. La biomasse au début des périodes expérimentales était respectivement de 5404 , 3533 et $1633 \mathrm{~kg}$ de M.S./ha. Les bols œsophagiens et les fèces ont été régulièrement récoltés à $8 \mathrm{~h} 30$ et 16 h 30 pendant sept jours. Les échantillons ont été immédiatement pesés et congelés en vue de leur lyophilisation.

Dans les 2 expériences, l'azote total a été déterminé par la méthode de Kjeldahl et la lignine par celle de Christian (1971) pour qui elle est le résidu de l'action

(1) Travaux subsidiés par le Fonds de la Recherche Fondamentale Collective Convention $n^{\circ} 2.4542 .84$. 
successive de $\mathrm{H}_{2} \mathrm{SO}_{4}$ à $72 \%$ et d'une solution à $2 \%$ d'un détergent acide pendant 2 heures.

Résultats et discussion. Les taux de récupération fécale de la lignine sont systématiquement supérieurs à 100 (tabl. 1). Les taux nettement excédentaires pour les deux herbes les moins lignifiées doivent vraisemblablement être attribués à l'obtention d'une lignine fécale particulièrement souillée. Les teneurs en azote beaucoup plus élevées de cette lignine (14 et $8 \%$ respectivement pour les raygrass fauchés les 22/04 et 09/06) nous font préférer cette hypothèse à celle d'une sous-estimation de la lignine alimentaire. Toutefois, une solubilisation partielle de la lignine de l'herbe ne doit pas être exclue.

TABL. 1. - Composition des fourrages (\% de la M.S.), taux de récupération fécale de la lignine (T.R.F.L.) et digestibilité de la matière sèche (d M.S. en \%) mesurée ou estimée à partir de la ligninemarqueur.

\begin{tabular}{|c|c|c|c|c|c|}
\hline \multirow{2}{*}{$\begin{array}{l}\text { Fourrage } \\
\text { Date de récolte }\end{array}$} & \multicolumn{2}{|c|}{ Ray-grass } & \multicolumn{3}{|c|}{ Herbe de prairie } \\
\hline & $22 / 04 / 80$ & $09 / 06 / 80$ & $27 / 05 / 81$ & $10 / 06 / 81$ & $09 / 09 / 80$ \\
\hline Fourrage M.A.T. & 21,6 & 6,4 & 11,9 & 12,0 & 14,8 \\
\hline Lignine & 0,63 & 4,1 & 1,9 & 2,9 & 1,6 \\
\hline T.R.F.L. & 128,7 & 105,5 & 106,4 & 109,3 & 126,4 \\
\hline d M.S. bilan & 79,1 & 63,2 & 73,8 & 71,4 & 69,1 \\
\hline lignine-marqueur & 83,1 & 64,9 & 75,6 & 72,9 & 75,3 \\
\hline
\end{tabular}

A partir des valeurs individuelles obtenues au cours des bilans mentionnés cidessus, nous avons calculé les relations suivantes permettant de déterminer la d M.S. (Y) à partir des teneurs en lignine des fèces $\left(L_{F}\right)$ ou à partir des teneurs en azote fécal $\left(N_{F}\right): Y=-1,446 L_{F}+83,249 ; r=-0,79, n=21, s_{y x}=6,12$ $Y=5,381 N_{F}+58,260 ; r=0,89, n=21, s_{y x}=4,50$

Ces équations ont été appliquées dans le cadre de l'expérience 2 . On observe une excellente concordance entre les valeurs obtenues par ces deux relations et celles calculées en utilisant la lignine comme marqueur indigestible interne (tabl. 2).

TABL. 2. - Composition (\% de la M.S.) de l'herbe (H) et des bols cesophagiens (B.O.) et digestibilité de la matière sèche $(\%)$ estimée selon diverses méthodes.

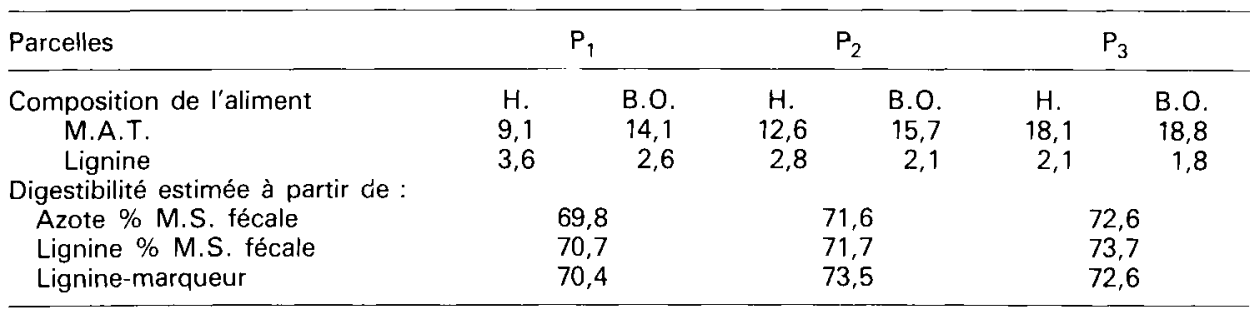

Ces résultats justifient la poursuite des travaux; une attention particulière sera accordée à l'influence d'une supplémentation en aliments concentrés.

Christian K. R., 1971. Detergent method for total lignin in herbage. Fie/d Stat. Rec. Div. PI. Ind. CSIRO (Aust.), 10, 29-34.

Frère P., Dubois J. P., François E., Théwis A., 1981. The use of lignin determined by the method of Christian for predicting dry matter digestibility of temperate forages, II-24. 32nd annu. Meet. $E A A P, 31$ Aug.-3 Sept. Zagreb (Yugoslavia). 